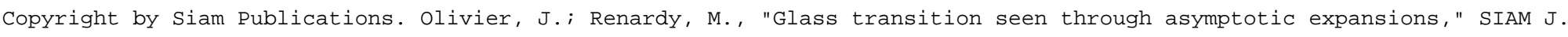
Appl. Math., 71(4), 1144-1167, (2011). DOI: 10.1137/100800725

\title{
GLASS TRANSITION SEEN THROUGH ASYMPTOTIC EXPANSIONS*
}

\author{
JULIEN OLIVIER ${ }^{\dagger}$ AND MICHAEL RENARDY ${ }^{\ddagger}$
}

\begin{abstract}
Soft glassy materials exhibit the so-called glassy transition, which means that the behavior of the model at a low shear rate changes when a certain parameter (which we call the glass parameter) crosses a critical value. This behavior goes from a Newtonian behavior to a HerschelBulkley behavior through a power-law-type behavior at the transition point. In a previous paper we rigorously proved that the Hébraud-Lequeux model, a Fokker-Planck-like description of soft glassy material, exhibits such a glass transition. But the method we used was very specific to the one-dimensional setting of the model, and as a preparation for generalizing this model to take into account multidimensional situations, we look for another technique to study the glass transition of this type of model. In this paper we shall use matched asymptotic expansions for such a study. The difficulties encountered when using asymptotic expansions for the Hébraud-Lequeux model are that multiple ansaetze have to be used, even though the initial model is unique, due to the glass transition. We shall delineate the various regimes and give a rigorous justification of the expansion by means of an implicit function argument. The use of a two parameter expansion plays a crucial role in elucidating the reasons for the scalings which occur.
\end{abstract}

Key words. glass transition, boundary layers, implicit functions, linear perturbation

AMS subject classifications. 34E10, 76A05

DOI. $10.1137 / 100800725$

1. Introduction. Soft glassy materials are complex fluids which by definition exhibit a transition when a parameter goes through a critical value. This behavior is very similar to that of classical glasses, in which case the parameter is simply the temperature of the glass. The class of materials we are interested in is formed of the so-called granular glasses. A granular glass is composed of "particles" dispersed in a Newtonian fluid: this is the case for suspensions of solid particles and for emulsions which are "particles" of a fluid dispersed in another immiscible fluid. In this setting the parameter is related to the concentration of particles: as long as the particles are not too concentrated, the behavior of the material is Newtonian. But when the concentration reaches a certain value, the particles are so packed that the structure has to break before the material can flow. This leads to a stress threshold behavior similar to Herschel-Bulkley models.

One model available in the literature to describe this kind of phenomenon is the one introduced by Hébraud and Lequeux in [11]. At the beginning, there was a kinetictype model for real glasses which was proposed by Bouchaud, Comtet, and Monthus in [2]. Then considering the aforementioned analogy between soft glasses and real glasses, a model was designed by Sollich et al. in [15] for soft glasses. Finally, the Hébraud-Lequeux model (HL) was introduced as a simpler model taking the previous ideas into account.

*Received by the editors July 1, 2010; accepted for publication (in revised form) May 2, 2011; published electronically July 21, 2011. This research was supported by ANR project Maniphyc ANR08-SYSC-010 and by the National Science Foundation under grant DMS-1008426.

http://www.siam.org/journals/siap/71-4/80072.html

${ }^{\dagger}$ Lama UMR 5127 CNRS, Université de Savoie, 73376 Le Bourget-du-Lac Cedex, France (julien. olivier@univ-savoie.fr).

${ }^{\ddagger}$ Department of Mathematics, Virginia Tech, Blacksburg, VA 24061-0123 (renardym@math.vt. edu). 
In a previous paper [13], we mathematically proved that HL describes the glass transition, as was announced in Hébraud's Ph.D. thesis [10]. This work relied on analytical computations of the solutions of the model, which was possible because HL was designed to describe simple shear flow and is thus, from a mathematical point of view, a one-dimensional problem governed by an ODE. Thus all the computations can be done "by hand" (note that for given $y$ and $\phi,(1.1)$ below is just an ODE with piecewise constant coefficients, which can be solved explicitly). But in an upcoming work we intend to extend the model to multidimensional situations and thus study a model similar to HL but set on several dimensions of space. This means that analytical solutions are not available anymore. This led us to search for other tools to prove the same results, techniques that would be more easily generalized to multidimensional situations. The tool we found to be appropriate is asymptotic expansion.

Let us introduce the model and our notation. We are interested in the stationary dimensionless version of HL. Here the unknown is $p$, which is a probability density on the stress space. The stress variable will always be denoted $\sigma$. The small parameter is $y$. It is a dimensionless shear rate and is chosen to be nonnegative. The glass parameter is denoted by $\mu$. Finally, the model uses a function $\phi$ of $y$ called the fluidity. Then the model reads

$$
\left\{\begin{array}{l}
-\phi(y) \partial_{\sigma}^{2} p+y \partial_{\sigma} p+\mathbf{1}_{[-1,1]^{c}} p=\frac{\phi(y)}{\mu} \delta_{0} \\
p \in \mathrm{H}^{1}(\mathbf{R}) \\
p( \pm \infty)=0 \\
p \geq 0 \\
\int_{\mathbf{R}} p(\sigma) \mathrm{d} \sigma=1 .
\end{array}\right.
$$

Here $\mathbf{1}_{[-1,1]^{c}}$ denotes the characteristic function of the complement of the interval $[-1,1]$, and $\delta_{0}$ is the delta function located at the origin. The fluidity is not explicitly defined. It comes from the constraint on the integral of $p$. Indeed, if instead of $\phi(y)$ in the PDE we had a given constant $\Gamma$, then solving the boundary value problem is a simple matter. But since we have in addition the integral constraint, there is only one $\Gamma$ that allows for solving the equation and the integral constraint simultaneously when $y>0$. See Cancès, Catto, and Gati [4] or [13] for further details on this question. On the other hand, by integrating the equation one has the following connection between $p$ and $\phi$ :

$$
\frac{\phi}{\mu}=\int_{|\sigma|>1} p(\sigma) \mathrm{d} \sigma
$$

Finally, to connect more specifically to the physics of the phenomenon we are trying to model, we introduce the macroscopic stress

$$
\tau=\int_{\mathbf{R}} \sigma p(\sigma) \mathrm{d} \sigma
$$

Then $\tau$ is only a function of $y$. In this setting the glass transition occurs at the critical value $1 / 2$ : when $\mu>1 / 2$ the behavior is Newtonian, that is, $\tau \sim \eta y$, when $\mu=1 / 2$ we will have a power-law fluid with exponent $1 / 5$, that is, $\tau \sim y^{1 / 5}$, and finally, when $\mu<1 / 2$ we obtain a Herschel-Bulkley fluid with exponent $1 / 2$, that is 
to say, $\tau \sim \tau_{0}+A \sqrt{y}$, where $\tau_{0}>0$ is called the dynamic yield stress. We note that the asymptotic expansions for $p$ translate in a straightforward manner to expansions for $\tau$, as long as we show convergence in a function space that embeds into a space for which $p \mapsto \int \sigma p$ is a continuous linear form.

The paper is organized as follows: In section 2 we introduce the physical background of the model and the motivation for the equations. In section 3 we discuss the limiting problem for $y=0$, which provides the foundation for the asymptotic analysis. Our main result is stated in section 4. Section 5 is devoted to the derivation of the asymptotic hierarchy. We explain how to find the appropriate scales of expansions and the sizes of the relevant boundary layers. In section 6 we justify the formal expansions by an implicit function argument. In doing so, we find it advantageous to introduce a priori two small parameters $a=y / \phi(y)$ and $b=\sqrt{\phi(y)}$. This allows us to reduce our problem to a single equation of the form $F(\mu, a, b)=0$. The behavior of $\phi$ as a function of $y$ arises naturally from the analysis of this function.

\section{Physical background.}

2.1. Description. In this section we shall review the physical features behind the Hébraud-Lequeux model. The model describes what are called soft glassy materials. These materials are amorphous. For example, consider an emulsion, that is, a material consisting of bubbles of one liquid plunged into another liquid, and let us imagine that the bubbles do not coalesce. Then the disposition of the bubbles can be very complicated and does not follow any particular order. The rheology of these materials needs to take into account some of the complexity in the material. Indeed, these kinds of materials are sensitive to what can be called the mechanical noise: because of the composition of the material, what happens to the bubbles at some place can have mechanical repercussions throughout the material. Nevertheless, this noise must be taken into account in a manageable way. In the model by Bouchaud, Comtet, and Monthus [2] or in Sollich et al. [15] the noise is taken into account a priori as a characteristic quantity of the material. In HL this is done by using a statistical approach which is simple but captures the basic physics of a typical soft glassy material. The noise can then be approached by mechanical considerations and is not "hidden" in the model. Only the intensity of the noise is characteristic of the material.

The model is restricted to simple shear flows. In classical rheology, when an "elementary" block of material undergoes a certain shear rate $\dot{\gamma}$, the continuous medium has a change in its stress $\sigma$ to adapt to the shear rate so that the stress and the shear rate are always linked. The nature of this link defines the rheological properties of the material. One can then study a macroscopic sample (assuming the shear rate is constant throughout the sample) by mentally dividing it into "elementary" blocks. But for microscopically inhomogeneous materials like suspensions or emulsions this procedure is not relevant since elementary blocks are in all kinds of states.

HL acknowledges this difficulty by, in a sense, attributing a distribution of stresses to the "elementary" blocks for a given macroscopic shear rate. More accurately, the model describes a typical "elementary" block by giving it a distribution of stress $p$ for a given shear rate that accounts for the distribution of stresses of all the "elementary" blocks (not typical ones but real ones) of a macroscopic sample. HL gives the evolution of this distribution in time. The quantity $p(t, \sigma) \mathrm{d} \sigma$ can be understood as the proportion of "elementary" blocks of a macroscopic sample that are in the stress state $\sigma$ (with margin $\mathrm{d} \sigma$ ) at time $t$. It is consequently a probability density. The stress of the macroscopic sample at time $t$, which we denote by $\tau(t)$, is the average of all the 
contributions of the "elementary" blocks and is therefore given by the equation

$$
\tau(t)=\int_{\sigma \in \mathbf{R}} \sigma p(t, \sigma) \mathrm{d} \sigma .
$$

We note that formulating the model in terms of only one stress component is an oversimplification. Even if the flow is restricted to parallel shear, stress is a tensor with several components, and all of them have a statistical distribution. The formulation and analysis of such models will be addressed in future work. Even in the case of simple shear flow, they do not simply reduce to the original HL.

Let us now give the evolution equation of $p$. Suppose that at time $t$ the shear rate throughout the sample is $\dot{\gamma}(t)$. Then we have

$$
\left.\partial_{t} p(t, \sigma)=-G_{0} \dot{\gamma}(t) \partial_{\sigma} p(t, \sigma)-\frac{1}{T_{0}} \mathbf{1}(\sigma)_{\left[-\sigma_{c}, \sigma_{c}\right]}\right]^{c} p(t, \sigma)+\Gamma(p)(t) \delta_{0}(\sigma)+\alpha \Gamma(p)(t) \partial_{\sigma}^{2} p(t, \sigma),
$$

where $G_{0}, \sigma_{c}, T_{0}$, and $\alpha$ are material constants respectively called elastic modulus, stress threshold, relaxation time, and fragility. The term $\mathbf{1}_{\left[-\sigma_{c}, \sigma_{c}\right]^{c}}$ designates the characteristic function of the complement of the segment $\left[-\sigma_{c}, \sigma_{c}\right]$ in the real line. The term $\Gamma(p)(t)$ is called the fluidity and depends on the state of the sample in the following way:

$$
\Gamma(p)(t)=\frac{1}{T_{0}} \int_{|\sigma|>\sigma_{c}} p(t, \sigma) \mathrm{d} \sigma .
$$

2.2. Explanations of the terms of the equation. Let us now explain heuristically how the equation is motivated.

We take a macroscopic sample of $N$ "elementary" blocks and for each of these blocks we denote $\Sigma_{i}(t)$ as its stress. We say that under the shear rate $\dot{\gamma}(t)$ the stress of an "elementary" block will evolve because of three effects:

Elastic gain. The first possibility is that the particular block behaves elastically, which means that its stress will evolve linearly in time, as prescribed by Hooke's law of linear elasticity:

$$
\Sigma_{i}(t+\mathrm{d} t)-\Sigma_{i}(t)=G_{0} \dot{\gamma}(t) \mathrm{d} t .
$$

Physically, we could say that the local structure withstands the shear by elastically increasing its stress. For an emulsion it could correspond to bubbles deforming to take in the shear rate. Of course, the actual source of elastic forces in an emulsion is the surface tension on the interfaces, so the assumption of linear Hookean elasticity is a rather crude simplification.

Relaxation toward 0 . When $\left|\Sigma_{i}\right|$ is larger than a maximal value $\sigma_{c}$, the local structure cannot bear the shear and locally breaks. By doing this the stress is released, and we model this effect by an exponential decay toward 0 with rate $T_{0}$. For an emulsion this would be, for example, a bubble that cannot deform any longer and moves away from its position to find a place where it would be less deformed and thus less stressed. We call this a relaxation event. Note that the fluidity is thus the time rate of relaxation events.

Rearrangements. When the structure breaks, it induces rearrangements on neighboring blocks. One can think, for example, that, in the case of an emulsion, when a bubble moves away because of the relaxation event, it lets its place to another bubble, which, in turn, lets its place to another one, and so on. We model this on a typical 
"elementary" block by adding a Brownian motion that accounts for the contribution of all the rearrangements around it. Of course the more relaxation events there are, the more rearrangements there can be, and the more this effect will be important. So the intensity of the Brownian motion will be proportional to the total number of relaxation events, and we call $\alpha / T_{0}$ the proportionality constant. When $\alpha$ is small, there needs to be a lot of relaxation events for the rearrangement to be perceived, and, on the contrary, if $\alpha$ is large, then a few relaxation events will induce lots of rearrangements everywhere. This can be interpreted as follows: rearrangements are by nature local (in space). If $\alpha$ is small, this means that the rearrangements will take place on a very small area around the relaxation event. So, for a given block, the extra stress due to rearrangement will be small because the block does not see the blocks that are far from it. On the other hand, if $\alpha$ is large, then even a few relaxation events will have consequences on a lot of blocks even far from the origin. In this interpretation, $\alpha$ is a measure of the fragility of the structure: when $\alpha$ is small, a break somewhere in the structure will have little repercussion, which means that the structure is robust. On the other hand, if $\alpha$ is large, then a small break at some place will induce a lot of breaks throughout the structure, and the structure can indeed be called fragile.

2.3. Dimensionless equation. It is usually easier to analyze models such as (2.2) by rescaling variables. To do that we need to choose a scale for all the variables. For the stress variable, we choose the scale given by the stress threshold $\sigma_{c}$, so we set $\sigma^{\prime}=\sigma / \sigma_{c}$. The relaxation time $T_{0}$ provides a time scale.

Since $p$ is a stress density, its dimension is the inverse of a stress. It is thus natural to introduce $p^{\prime}\left(t^{\prime}, \sigma^{\prime}\right)=\sigma_{c} p\left(t^{\prime} T, \sigma^{\prime} \sigma_{c}\right)$. Note also that when changing variables we have $\delta_{0}(\sigma)=\left(1 / \sigma_{c}\right) \delta_{0}\left(\sigma / \sigma_{c}\right)$. One can check that $p^{\prime}$ follows the following equation:

$$
\begin{aligned}
\partial_{t^{\prime}} p^{\prime}\left(t^{\prime}, \sigma^{\prime}\right)=-\frac{G_{0}}{\sigma_{c}}\left(T_{0} \dot{\gamma}\left(t^{\prime} T_{0}\right)\right) \partial_{\sigma^{\prime}} & p^{\prime}\left(t^{\prime}, \sigma^{\prime}\right)-\mathbf{1}\left(\sigma^{\prime}\right)_{[-1,1]^{c}} p^{\prime}\left(t^{\prime}, \sigma^{\prime}\right) \\
& +\Gamma^{\prime}\left(p^{\prime}\right)\left(t^{\prime}\right) \delta_{0}\left(\sigma^{\prime}\right)+\frac{\alpha}{\sigma_{c}^{2}} \Gamma^{\prime}\left(p^{\prime}\right)\left(t^{\prime}\right) \partial_{\sigma^{\prime}}^{2} p^{\prime}\left(t^{\prime}, \sigma^{\prime}\right),
\end{aligned}
$$

where

$$
\Gamma^{\prime}\left(p^{\prime}\right)\left(t^{\prime}\right)=\int_{\left|\sigma^{\prime}\right|>1} p^{\prime}\left(t^{\prime}, \sigma^{\prime}\right) \mathrm{d} \sigma^{\prime}
$$

We also have, by this change of variables,

$$
\int_{\sigma^{\prime} \in \mathbf{R}} p^{\prime}\left(t^{\prime}, \sigma^{\prime}\right) \mathrm{d} \sigma^{\prime}=1
$$

We thus define two dimensionless numbers: $\lambda=G_{0} / \sigma_{c}$, and what we call the glass parameter, $\mu=\alpha / \sigma_{c}^{2}$. In what follows, we drop the primes in the dependent and independent variables.

In this paper, we focus on the stationary equation; i.e., all variables are independent of time. This results in the following model (in dimensionless form):

$$
0=-\lambda\left(T_{0} \dot{\gamma}\right) \partial_{\sigma} p(\sigma)-\mathbf{1}(\sigma)_{[-1,1]^{c}} p(\sigma)+\Gamma(p) \delta_{0}(\sigma)+\mu \Gamma(p)(t) \partial_{\sigma}^{2} p(\sigma) .
$$


We note that the shear rate appears only in the combination $\lambda T_{0} \dot{\gamma}$. This dimensionless combination is referred to as a Weissenberg number, and it is what we have denoted by $y$ in our analysis. Also, we use $\phi=\mu \Gamma$ instead of $\Gamma$, which is the diffusion coefficient of the equation. That is how we find (1.1) from (2.2).

2.4. Heuristic considerations. The essential dimensionless parameter in the equations is therefore the glass transition parameter $\mu$. Let us assess the relevance of this parameter. If $\mu$ is small, we have elastic behavior until $|\sigma|$ exceeds 1 , and then we have relaxation. The result of this is a yield stress behavior; since a critical stress must be reached before relaxation can occur, the fluid has to overcome this critical stress to flow, no matter how small the shear rate is. On the other hand, if $\mu$ is large, the random effect of rearrangements can lead to stresses exceeding the critical stress. The distribution of microstresses becomes broad, and relaxation always occurs. In this scenario, the yield stress disappears, and the fluid can flow at any shear rate. Below, we shall specifically quantify these phenomena.

3. Behavior at main order. In this section we shall briefly discuss the "fundamental" solutions that govern the limiting behavior of system (1.1) in the limit $y=0$. As the glass transition implies, there are three possible regimes. The first one is the Newtonian regime. In this regime the material constantly undergoes breaks and rearrangements (a process sometimes known as rejuvenation; see [14]), even in the limit of vanishing shear rate. We thus expect at the main order a stationary stress distribution which is nonzero for $|\sigma|>1$. The specific expression is obtained by setting $y=0$ in the governing equation, while we expect a nonzero limit (which is to be determined) for $\phi(y)$. In what follows we use the notation

$$
q=\left.p\right|_{]-1,1[}, \quad r_{g}=\left.p\right|_{]-\infty,-1[}, \quad \quad r_{d}=\left.p\right|_{] 1,+\infty[},
$$

and we use $\bar{Q}^{0}, \bar{R}_{g}^{0}$, and $\bar{R}_{d}^{0}$ for the leading order of $q, r_{g}$, and $r_{d}$, respectively. In the Newtonian regime the stationary distribution at main order is governed by the following equations:

$$
\left\{\begin{array}{l}
-\mu \bar{c}_{0} \partial_{\sigma}^{2} \bar{Q}^{0}=\bar{c}_{0} \delta_{0} \\
-\mu \bar{c}_{0} \partial_{\sigma}^{2} \bar{R}_{g}^{0}+\bar{R}_{g}^{0}=0 \\
-\mu \bar{c}_{0} \partial_{\sigma}^{2} \bar{R}_{d}^{0}+\bar{R}_{d}^{0}=0 \\
\bar{Q}^{0}(-1)=\bar{R}_{g}^{0}(-1) \\
\bar{Q}^{0}(1)=\bar{R}_{d}^{0}(1) \\
\partial_{\sigma} \bar{Q}^{0}(-1)=\partial_{\sigma} \bar{R}_{g}^{0}(-1) \\
\partial_{\sigma} \bar{Q}^{0}(1)=\partial_{\sigma} \bar{R}_{d}^{0}(1) \\
\int_{-1}^{1} \bar{Q}^{0}(\sigma) \mathrm{d} \sigma+\bar{c}_{0}=1 \\
\bar{c}_{0} \neq 0
\end{array}\right.
$$

This problem is simply (1.1) with $y=0$ and $\phi / \mu=\bar{c}_{0}$ when split into the three intervals $]-\infty,-1[]-1,,1[$, and $] 1,+\infty[$. The explicit solution of this system, which 


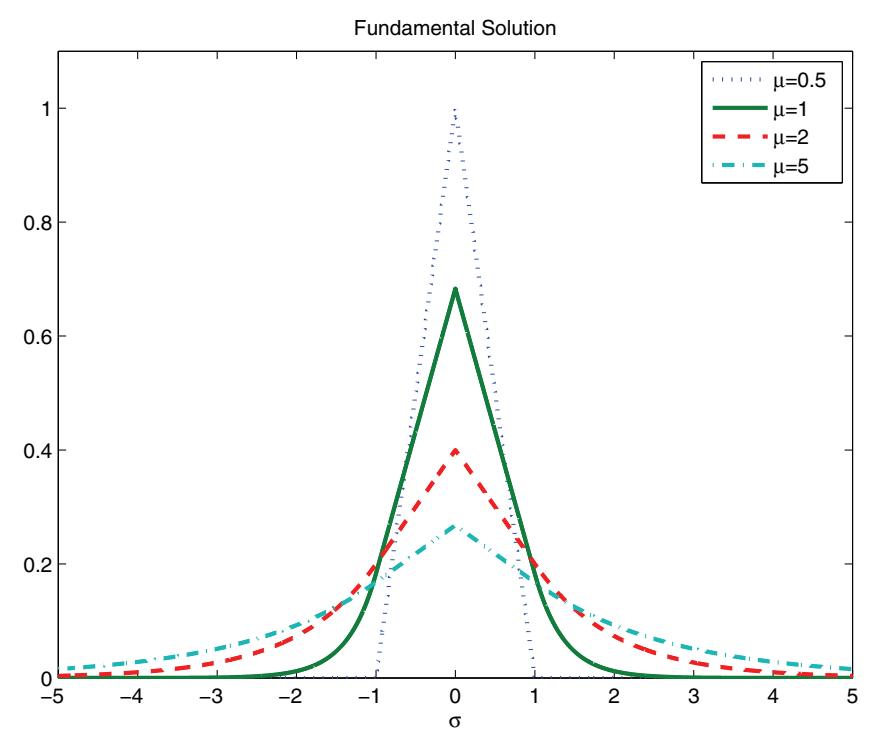

FIG. 3.1. Examples of fundamental solutions for $\mu \geq 1 / 2$.

can be found in $[4,13]$, is

$$
\begin{aligned}
& \bar{Q}^{0}(\sigma)=\left\{\begin{array}{l}
\frac{\sigma+1}{2 \mu}+\frac{1}{2} \sqrt{\frac{\bar{c}_{0}}{\mu}} \quad \text { if } \sigma<0, \\
\frac{1-\sigma}{2 \mu}+\frac{1}{2} \sqrt{\frac{\bar{c}_{0}}{\mu}} \quad \text { if } \sigma>0,
\end{array}\right. \\
& \bar{R}_{g}^{0}(\sigma)=\frac{1}{2} \sqrt{\frac{\bar{c}_{0}}{\mu}} \exp \left(\frac{\sigma+1}{\sqrt{\bar{c}_{0} \mu}}\right), \\
& \bar{R}_{d}^{0}(\sigma)=\frac{1}{2} \sqrt{\frac{\bar{c}_{0}}{\mu}} \exp \left(-\frac{\sigma-1}{\sqrt{\bar{c}_{0} \mu}}\right),
\end{aligned}
$$

where $\bar{c}_{0}$ satisfies the equation (which is a statement of the integral constraint)

$$
\bar{c}_{0}+\sqrt{\frac{\bar{c}_{0}}{\mu}}+\frac{1}{2 \mu}=1
$$

and thus has the following solution:

$$
\bar{c}_{0}=1-\frac{1}{2 \mu} \sqrt{4 \mu-1}
$$

Fundamental solutions for several values of $\mu \geq 1 / 2$ are shown in Figure 3.1. As announced, $\bar{R}_{g}^{0}>0$ and $\bar{R}_{d}^{0}>0$, and since the function obtained by gluing the previous pieces together is even, there is no stored stress in this fundamental problem. Indeed, applying formula (1.3) to this function gives 0 . Finally, we remark that if $\mu \leq 1 / 2$, then (3.3) does not have a positive solution, and that is why problem (3.1) cannot be the fundamental problem of (1.1) when $\mu \leq 1 / 2$.

The second regime is the jammed regime. In this regime, the effect of rearrangements is not strong enough to take the local stress beyond the threshold. At the 
limit, all the blocks are trapped in states that no longer allow relaxation events. As $y$ becomes smaller, the elastic mechanism becomes weaker and cannot bring the stress high enough for relaxations to occur. In the end the stationary distribution of stress reflects that fact since $\bar{R}_{g}^{0}=\bar{R}_{d}^{0}=0$. However, our analysis can give information on the inner distribution of stress "at the limit." This means that if we knew that a soft glassy material, in the jammed state, had been put to rest through a sequence of decreasing shear rate, with enough time to reach the stationary state at each step, then we would be able to compute the complete distribution of stress in the material. Note that without the history of the preparation of the material sample, we would not be able to distinguish between any distributions of stress which do not allow relaxation events. Indeed, if we set $y=\phi(y)=0$, then all the terms in (1.1) vanish in the inner region, and we obtain absolutely no information. We can, however, obtain a meaningful limit by assuming that $\phi(y)$ is proportional to $y$ and then dividing the equation by $y$. If we set $\phi(y)=\mu c_{1} y$, we obtain the following problem for $\bar{Q}^{0}$ :

$$
\left\{\begin{array}{l}
-\mu c_{1} \partial_{\sigma}^{2} \bar{Q}^{0}+\partial_{\sigma} \bar{Q}^{0}=c_{1} \delta_{0} \\
\bar{Q}^{0}( \pm 1)=0 \\
\int_{-1}^{1} \bar{Q}^{0}(\sigma) \mathrm{d} \sigma=1 \\
c_{1} \neq 0
\end{array}\right.
$$

For this system, $\bar{Q}^{0}$ can be computed in terms of $c_{1}$, and the integral constraint gives us an equation on $c_{1}$. Namely, we have

$$
\bar{Q}^{0}(\sigma)= \begin{cases}\frac{c_{1}\left(1-\exp \left(-\frac{1}{\mu c_{1}}\right)\right)}{\exp \left(\frac{1}{\mu c_{1}}\right)-\exp \left(-\frac{1}{\mu c_{1}}\right)}\left(\exp \left(\frac{\sigma+1}{\mu c_{1}}\right)-1\right) & \text { if } \sigma \in]-1,0[, \\ \frac{c_{1}\left(\exp \left(\frac{1}{\mu c_{1}}\right)-1\right)}{\exp \left(\frac{1}{\mu c_{1}}\right)-\exp \left(-\frac{1}{\mu c_{1}}\right)}\left(1-\exp \left(\frac{\sigma-1}{\mu c_{1}}\right)\right) & \text { if } \sigma \in] 0,1[,\end{cases}
$$

and $c_{1}$ is fixed by the following equation (which again is a statement of the integral constraint):

$$
c_{1} \tanh \left(\frac{1}{2 \mu c_{1}}\right)=1
$$

If we multiply this equation by $\mu$ and study $x \mapsto x \tanh (1 /(2 x))$, it is easy to see that it does not have a solution if $\mu \geq 1 / 2$, and that is why problem (3.4) cannot serve as a fundamental problem for this range of $\mu$. Finally, the solution is clearly not even, and using (1.3) on $\bar{Q}^{0}$ instead of $p$, one can compute a positive value $\tau_{0}$. This value is called the dynamic yield stress. Fundamental solutions for several values of $\mu \leq 1 / 2$ are shown in Figure 3.2.

The final regime is the transition regime which is in between the two previous 


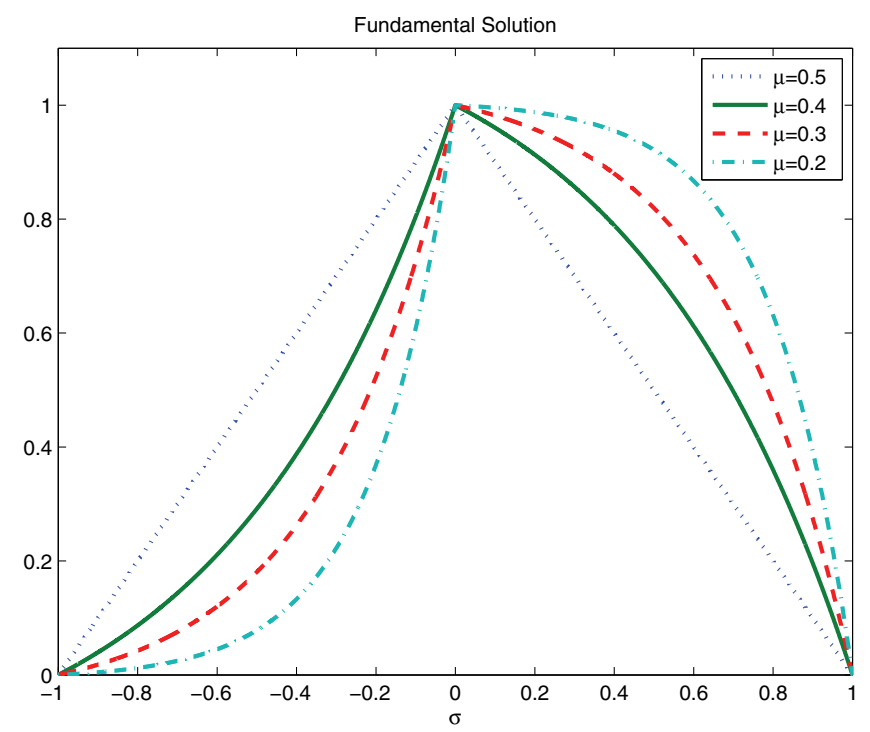

FIG. 3.2. Examples of fundamental solutions for $\mu \leq 1 / 2$.

regimes. Its governing equations would be

$$
\left\{\begin{array}{l}
-\mu \partial_{\sigma}^{2} \bar{Q}^{0}=\delta_{0} \\
\bar{Q}^{0}( \pm 1)=0 \\
\int_{-1}^{1} \bar{Q}^{0}(\sigma) \mathrm{d} \sigma=1 .
\end{array}\right.
$$

This limit is obtained under the assumption that $\phi(y) \rightarrow 0$, but $\phi(y) / y \rightarrow \infty$ as $y \rightarrow 0$. The exact behavior of $\phi(y)$ is not obtained at this level and can only be determined by considering higher orders in the expansion. This will be pursued further in section 5 .

However, it is easily seen that system (3.7) can only be solved when $\mu=1 / 2$ by the function

$$
\bar{Q}^{0}(\sigma)= \begin{cases}\sigma+1 & \text { if } \sigma \in[-1,0], \\ 1-\sigma & \text { if } \sigma \in[0,1] .\end{cases}
$$

As we can see, this solution is really in between the two previous regimes: its support is $[-1,1]$, so it is in a jammed state as are the fundamental solutions of the the $\mu<1 / 2$ case. However, the function is even, as in the $\mu>1 / 2$ case, and as such does not store any stress.

4. Reformulation of the problem and main result. At the start of this study we were inspired by the similarity of the problem given by the system (1.1) to the problem of stationary Navier-Stokes equations with a penalization term to take into account an obstacle in viscous flows studied by Angot, Bruneau, and Fabrie [1], Carbou and Fabrie [6], or Carbou [5] in the context of porous materials, or the problem of wave equations with a penalization term studied by Fornet and Guès [9] and Fornet 
$[8,7]$. This encouraged us to see whether the same methods could be adapted to our problem. In the HL setting, the obstacle would be the exterior of the $[-1,1]$ interval.

The first thing to do is to separate what happens inside and outside the obstacle. This means we rewrite (1.1) with unknowns

$$
q=\left.p\right|_{]-1,1[}, \quad r=\left.p\right|_{[-1,1]^{c}}
$$

and obtain

$$
\begin{array}{lr}
-\phi(y) \partial_{\sigma}^{2} q+y \partial_{\sigma} q=\frac{\phi(y)}{\mu} \delta_{0} & \text { in }]-1,1[, \\
-\phi(y) \partial_{\sigma}^{2} r+y \partial_{\sigma} r+r=0 & \text { in }[-1,1]^{c}, \\
r( \pm \infty)=0, & \\
q \geq 0, & \\
r \geq 0, & \\
r( \pm 1)=q( \pm 1), & \\
\partial_{\sigma} r( \pm 1)=\partial_{\sigma} q( \pm 1), & \\
\int_{|\sigma|>1} r(\sigma) \mathrm{d} \sigma+\int_{-1}^{1} q(\sigma) \mathrm{d} \sigma=1 . &
\end{array}
$$

We refer to (4.1f) and (4.1g) as the transmission conditions and (4.1h) as the integral constraint.

Now our main result can be stated as follows.

THEOREM 4.1. The solution of $(4.1 \mathrm{a})-(4.1 \mathrm{~h})$, for small $y$, can be expanded in a convergent series whose terms can be described in terms of boundary layers. Moreover, the expansion changes if the parameter $\mu$ changes, which leads more precisely to the following discussion.

If $\mu>1 / 2$, there are no boundary layer terms ( $\bar{Q}$ and $\bar{R}$ are functions of $\sigma$ ), and we have

$$
\begin{aligned}
& q=\bar{Q}^{0}+y \bar{Q}^{1}+y^{2} \bar{Q}^{2}+\cdots, \\
& r=\bar{R}^{0}+y \bar{R}^{1}+y^{2} \bar{R}^{2}+\cdots,
\end{aligned}
$$

and consequently

$$
\frac{\phi}{\mu}=\bar{c}_{0}+\bar{c}_{1} y+\bar{c}_{2} y^{2}+\cdots
$$

If $\mu<1 / 2$, the boundary layer is of size $y^{1 / 2}$ and

$$
\begin{aligned}
& q=\bar{Q}^{0}+y^{1 / 2} \bar{Q}^{1}+y \bar{Q}^{2}+\cdots, \\
& r=\sqrt{y} R^{1}+y R^{2}+\cdots,
\end{aligned}
$$

and consequently

$$
\frac{\phi}{\mu}=c_{1} y+c_{2} y^{3 / 2}+\cdots,
$$

with $R^{k}$ depending on $(|\sigma|-1) / y^{1 / 2}$.

Copyright $@$ by SIAM. Unauthorized reproduction of this article is prohibited. 
If $\mu=1 / 2$, the boundary layer is of size $y^{2 / 5}$ and

$$
\begin{aligned}
& q=\bar{Q}^{0}+y^{1 / 5} \bar{Q}^{1}+y^{2 / 5} \bar{Q}^{2}+\cdots, \\
& r=y^{2 / 5} R^{2}+y^{3 / 5} R^{3}+\cdots,
\end{aligned}
$$

and consequently

$$
\frac{\phi}{\mu}=c_{2} y^{4 / 5}+c_{2} y+\cdots,
$$

with $R^{k}$ depending on $(|\sigma|-1) / y^{2 / 5}$.

Using the previous theorem, the following result related to the macroscopic stress may be deduced.

COROLlary 4.2. The previous expansions converge in a space in which the application $p \mapsto \int_{\mathbf{R}} \sigma p$ is a continuous linear form. Thus by a simple integration we have the following expansions.

If $\mu>1 / 2$, the stress expands as

$$
\tau=\eta_{0} y+\eta_{1} y^{2}+\cdots .
$$

If $\mu<1 / 2$, the stress expands as

$$
\tau=\tau_{0}+A_{0} \sqrt{y}+\cdots .
$$

If $\mu=1 / 2$, the stress expands as

$$
\tau=B_{0} y^{1 / 5}+B_{1} y^{2 / 5}+\cdots .
$$

The various constants (which may be 0 except for the first one of those we have indicated) can be computed in terms of the profiles of the expansion of $q$ and $r$.

Section 5 is devoted to understanding from a formal point of view where the boundary layers come from, starting from an a priori unknown boundary layer expansion (meaning that we will prescribe neither the size of the boundary layer nor the scale of the expansion). We will then obtain the equations of the profile in each case. This method is fairly general and may be applied in multidimensional generalizations, and the equations of profile can be useful for numerical purposes.

Even though this section does not contain any proof of convergence, one could follow up with a convergence proof based on existence and uniqueness of the profiles and estimation of the remainder at a given order, in a manner similar to Boyer and Fabrie [3]). However, in section 6 we shall give a simpler proof which exploits the fact that the problem is more easily analyzed in a two parameter setting. In this setting, $a=y / \phi(y)$ and $b=\sqrt{\phi(y)}$ are treated a priori as independent parameters. It turns out that the solution of the differential equation is actually an analytic function of $a$ and $b$. The proof uses perturbation theory. One of the essential differences between the one-dimensional and multidimensional cases is that the limit $b \rightarrow 0$ becomes a singular perturbation problem in several dimensions. The perturbation argument would therefore become more complicated, and the solution would depend only smoothly, but not analytically, on $b$. In a second step, we analyze the remaining equation resulting from the integral constraint. This is a finite dimensional problem of the form $F(\mu, a, b)=0$. The implicit function theorem can be used to establish a relationship between $a$ and $b$ which naturally yields the expansions of Theorem 4.1. 
5. Derivation of the asymptotic hierarchy. In this section we shall show the formal computations that will be justified by the next section. The formal computations are of interest in their own right, since the appropriate ansatz is not obvious a priori. Of course the results described by Hébraud and Lequeux in [11] and proved in [13] were a powerful guide. Yet they do not give the actual ansatz. The transitional case $\mu=1 / 2$ was especially hard to devise.

5.1. Ansatz. To describe the boundary layer which lies in the exterior domain $[-1,1]^{c}$, we need the distance to the boundary $\{-1,1\}$, and we call this distance $\theta_{e}$, which is simply

$$
\theta_{e}(\sigma)=|\sigma|-1
$$

We make the following ansatz for $q$ and $r$ :

$$
\begin{aligned}
q(\sigma) & =\sum_{k=0}^{+\infty} y^{k / s} \bar{Q}^{k}(\sigma), \\
r(\sigma) & =\sum_{k=0}^{+\infty} y^{k / s} \bar{R}^{k}(\sigma)+y^{k / s} R^{k}\left(\operatorname{sign}(\sigma), \frac{\theta_{e}(\sigma)}{y^{l / s}}\right)
\end{aligned}
$$

which implies that $\phi / \mu$ has the following expansion in view of (1.2):

$$
\frac{\phi(y)}{\mu}=\sum_{k=0}^{+\infty} \tilde{c}_{k} y^{k / s}
$$

Here $l$ and $s$ are two integers satisfying $1 \leq l \leq s$. We have also introduced

$$
\tilde{c}_{k}= \begin{cases}\bar{c}_{k} & \text { if } 0 \leq k \leq l-1, \\ \bar{c}_{k}+c_{k-l} & \text { if } k \geq l,\end{cases}
$$

with

$$
\bar{c}_{k}=\int_{|\sigma|>1} \bar{R}(\sigma) \mathrm{d} \sigma, \quad c_{k}=\int_{0}^{+\infty} y^{(k+l) / s}\left[R^{k}(-1, z)+R^{k}(1, z)\right] \mathrm{d} z .
$$

We recall that in a boundary layer setting we have, from a formal point of view, the property

$$
\forall k \forall m \quad \lim _{z \rightarrow \infty}\left|\partial_{z}^{m} R^{k}( \pm 1, z)\right|=0 .
$$

In more detail, we have the following.

Proposition 5.1 (necessary form of the ansatz). The parameters in the previous ansatz in each case can only be as follows.

If $\mu>1 / 2$, we have $s=1$ and no boundary layer, which means $l$ is undefined and all the $R^{k}$ are zero.

If $\mu<1 / 2$, we have $s=2$ and $l=1$, which means that the boundary layer is of size $y^{1 / 2}$. In the exterior all the $\bar{R}^{k}$ are zero.

If $\mu=1 / 2$, we have $s=5$ and $l=2$, which means that the boundary layer is of size $y^{2 / 5}$ while the expansion is in powers of $y^{1 / 5}$. In the exterior all the $\bar{R}^{k}$ are again zero.

Copyright $@$ by SIAM. Unauthorized reproduction of this article is prohibited. 
What we imply by necessary conditions is that taking other parameters will rapidly lead to ill-posed problems for the profiles, even at the leading order. Another detail to note is that for $\mu<1 / 2$, for instance, one could take $s=4$ and $l=2$, but this would lead to the same expansion with a lot of coefficients (half, in fact) simply vanishing. We have indicated the choices of parameters that lead to the minimum of "trivially zero" terms.

Our interest in this proposition is that its proof gives a methodology for finding the size of the boundary layer when you have no a priori knowledge (from physics or elsewhere) to guide you. We of course have in mind our multidimensional generalization of HL for which we lack this kind of information.

Before we can prove this proposition, we need to derive the equations solved by the profiles.

5.2. Equations of profile. We now put these ansaetze into (4.1a)-(4.1h) and assemble the terms of the same formal order. We obtain the following hierarchy of equations.

Equation (4.1a). We put the ansatz (5.1a) and the ansatz (5.1c) into (4.1a):

$$
\begin{array}{ll}
0 \leq k \leq s-1: & -\mu \sum_{k^{\prime}=0}^{k} \tilde{c}_{k^{\prime}} \partial_{\sigma}^{2} \bar{Q}^{k-k^{\prime}}=\tilde{c}_{k} \delta_{0}, \\
s \leq k: & -\mu \sum_{k^{\prime}=0}^{k} \tilde{c}_{k^{\prime}} \partial_{\sigma}^{2} \bar{Q}^{k-k^{\prime}}+\partial_{\sigma} \bar{Q}^{k-s}=\tilde{c}_{k} \delta_{0} .
\end{array}
$$

Equation (4.1b). We put the ansatz (5.1b) and the ansatz (5.1c) into (4.1b). We can then separate in these equations the equations obeyed by the $\bar{R}^{k}$ and those obeyed by the $R^{k}$ by using the property stated in (5.3). We then obtain

$$
\begin{array}{ll}
-2 l \leq k \leq-1: & -\mu \sum_{k^{\prime}=0}^{k+2 l} \tilde{c}_{k^{\prime}} \partial_{z}^{2} R^{k+2 l-k^{\prime}}=0, \\
0 \leq k \leq s-l-1: & -\mu \sum_{k^{\prime}=0}^{k+2 l} \tilde{c}_{k^{\prime}} \partial_{z}^{2} R^{k+2 l-k^{\prime}}+R^{k}=0, \\
s-l \leq k: & -\mu \sum_{k^{\prime}=0}^{k+2 l} \tilde{c}_{k^{\prime}} \partial_{z}^{2} R^{k+2 l-k^{\prime}}+R^{k}=-\theta_{e}^{\prime} \partial_{z} R^{k-s+l}
\end{array}
$$

and

$$
\begin{array}{ll}
0 \leq k \leq s-1: & -\mu \sum_{k^{\prime}=0}^{k} \tilde{c}_{k^{\prime}} \partial_{\sigma}^{2} \bar{R}^{k-k^{\prime}}+\bar{R}^{k}=0, \\
s \leq k: & -\mu \sum_{k^{\prime}=0}^{k} \tilde{c}_{k^{\prime}} \partial_{z}^{2} \bar{R}^{k-k^{\prime}}+\bar{R}^{k}=-\partial_{\sigma} \bar{R}^{k-s} .
\end{array}
$$

Equation (4.1c). With (5.3) in mind, (4.1c) tells us only that

$$
0 \leq k: \quad \bar{R}^{k}( \pm \infty)=0 .
$$

Equation (4.1f). The continuity relation translates to

$$
0 \leq k: \quad \bar{Q}^{k}( \pm 1)=\bar{R}^{k}( \pm 1)+R^{k}( \pm 1,0) .
$$


Equation (4.1g). The continuity of the derivative translates to (given that $\theta_{e}^{\prime}$ is nonzero)

$$
\begin{array}{ll}
0 \leq k \leq l-1: & 0=\partial_{z} R^{k}( \pm 1,0) \\
0 \leq k: & \partial_{\sigma} \bar{Q}^{k}( \pm 1)=\partial_{\sigma} \bar{R}^{k}( \pm 1)+\theta_{e}^{\prime}( \pm 1) \partial_{z} R^{k+l}( \pm 1,0) .
\end{array}
$$

Equation (4.1h). Finally, we get from (4.1h) the following constraints for the profile:

$$
\begin{aligned}
& k=0: \quad \int_{-1}^{1} \bar{Q}^{0}(\sigma) \mathrm{d} \sigma+\frac{\tilde{c}_{0}}{\mu}=1, \\
& k>0: \quad \int_{-1}^{1} \bar{Q}^{k}(\sigma) \mathrm{d} \sigma+\frac{\tilde{c}_{k}}{\mu}=0 .
\end{aligned}
$$

Influence of (4.1d) and (4.1e). The two conditions (4.1d) and (4.1e) translate to the positivity of the lowest order term of the expansions (5.1a) and (5.1b). Moreover, by taking into account (5.2), this also means that the first nonzero $\tilde{c}_{k}$ must be positive.

5.3. Proof of Proposition 5.1. Now that we have the equations solved by the profiles, we can turn to the proof of Proposition 5.1. We break it down into several steps.

Lemma 5.2. If $\tilde{c}_{0} \neq 0$, then all the $R^{k}$ are necessarily zero (thus $l$ is undefined).

Proof. We argue by induction. Take $k=-2 l$ in (5.4b) and $k=0$ in (5.4f) and in (5.3). Then the problem solved by $R^{0}$ is

$$
\left\{\begin{aligned}
\forall z>0 \quad-\mu \tilde{c}_{0} \partial_{z}^{2} R^{0}( \pm 1, z) & =0 \\
-\partial_{z} R^{0}( \pm 1,0) & =0 \\
R^{0}( \pm 1,+\infty) & =0
\end{aligned}\right.
$$

whose only solution is $R^{0}=0$. Now suppose $R^{0}, \ldots, R^{p-1}$ are identically 0 for $1 \leq p$. Then take $k=-2 l+p$ in (5.4b). We find that $R^{p}$ satisfies the equation

$$
-\mu \tilde{c}_{0} \partial_{z}^{2} R^{p}=0
$$

i.e., $\partial_{z}^{2} R^{p}=0$. But since from (5.3) $\partial_{z} R^{p} \rightarrow 0$ as $z$ tends to infinity, we find $\partial_{z} R^{p}=0$ for all $z$. Then again $R^{p} \rightarrow 0$ at infinity and $R^{p}$ is identically 0 .

Now we look at the other case when $\tilde{c}_{0}=0$.

LEMma 5.3. If $\tilde{c}_{0}=0$, then necessarily

1. $\bar{R}^{k}$ is identically 0 (and thus so is $\bar{c}_{k}$ ) for all $k$,

2. $c_{1}, \ldots, c_{l-1}$ are 0 and $c_{l}$ cannot be 0 , and

3. $R^{0}, \ldots, R^{l-1}$ are all identically zero.

Proof. The first point is proved by induction. Taking (5.4c) for $k=0$ gives

$$
-\mu \tilde{c}_{0} \partial_{z}^{2} \bar{R}^{0}+\bar{R}^{0}=0
$$

and since $\tilde{c}_{0}=0$ we have $\bar{R}^{0}=0$. The induction is then clear since all profiles $\bar{R}^{k}$ will satisfy the same equation.

To prove the second point, introduce $m$, the index of the first nonzero $c_{k}$, and suppose $0 \leq m \leq l-1$. Then take (5.4b) with $k=m-l$, which is the first nontrivially satisfied equation for the $R^{k}$. Since $m \leq l-1$, we have $m-l \leq-1$. Provide for this 
equation the boundary condition given by (5.4f) with $k=0$ and the property (5.3), and this leads to the following problem for $R^{0}$ :

$$
\left\{\begin{aligned}
\forall z>0 \quad-\mu c_{m} \partial_{z}^{2} R^{0}( \pm 1, z) & =0 \\
-\partial_{z} R^{0}( \pm 1,0) & =0 \\
R^{0}( \pm 1,+\infty) & =0
\end{aligned}\right.
$$

which can only be satisfied by the null function. All following $R^{k}$ will then satisfy the same problem and be consequently 0 , and thus so is $R^{m}$. This is contradictory since $c_{m}$ is supposed to be nonzero and the integral of $R^{m}$.

Suppose now that $m \geq l+1$. Once again we argue by induction that all $R^{k}$ are identically 0 , which leads to a contradiction with $c_{m} \neq 0$. Take $k=0$ in (5.4b), and you find that $R^{0}=0$ because all the coefficients in the sum are 0 due to the fact that the first index for which $\tilde{c}_{k}$ is nonzero is $l+m$. Then suppose $R^{0}, \ldots, R^{p-1}$ are all identically zero, and take $k=p$ in (5.4b). When we look at

$$
\sum_{k^{\prime}=0}^{p+2 l} \tilde{c}_{k^{\prime}} \partial_{z}^{2} R^{p+2 l-k^{\prime}}
$$

we see that if $k^{\prime} \leq m+l-1$, then $\tilde{c}_{k^{\prime}}=0$, and if $k^{\prime} \geq m+l$, then $p+2 l-k^{\prime} \leq p-(m-l)$, and thus $\partial_{z}^{2} R^{p+2 l-k^{\prime}}=0$, so that the sum is in fact 0 . And if $p \geq 0$, then we can conclude $R^{p}=0$.

Finally, suppose $m=l$. By taking $k=0$ in (5.4b) and in (5.4f) and then using the fact that we have $\tilde{c}_{0}=\cdots=\tilde{c}_{2 l-1}=0$, we find that the problem solved by $R^{0}$ is

$$
\left\{\begin{aligned}
\forall z>0 \quad-\mu c_{l} \partial_{z}^{2} R^{0}( \pm 1, z)+R^{0}( \pm 1, z) & =0 \\
-\partial_{z} R^{0}( \pm 1,0) & =0 \\
R^{0}( \pm 1,+\infty) & =0
\end{aligned}\right.
$$

which once again can only be solved by the null function. Argue by induction to find that $R^{1}, \ldots, R^{l-1}$ all satisfy the same problem as $R^{0}$ and are then identically 0 .

Remark 1 . The fact that $\tilde{c}_{2 l}$ is the first nonzero term of the expansion of $\phi$ is not surprising since in the equation of $r$ you would want to balance the second order derivative with the zeroth order term to have an exponentially fast decay at infinity, and when the coefficient in front of the second order derivative is of order $y^{\alpha}$ this can only be achieved if the boundary layer size is $y^{\alpha / 2}$.

Now we complete the proof of the proposition.

LEMma 5.4. If $\tilde{c}_{0} \neq 0$, then the fundamental problem is well-posed if and only if $\mu>1 / 2$. In this case $s$ can be taken as one.

Proof. Now we can look at the problem satisfied by $\bar{Q}^{0}, \bar{R}^{0}$, and $\bar{c}_{0}=\tilde{c}_{0}$. We take $k=0$ in $(5.4 \mathrm{a})$ and in $(5.4 \mathrm{c})$ and $k=-2 l$ in $(5.4 \mathrm{~b})$ for the equations and complete with the transmission condition from (5.4e) and (5.4g) with $k=0$ and finally $k=0$ in $(5.4 \mathrm{~h})$. This leads to problem (3.1), whose solution is given by (3.2).

Now we show that for $k=1+n s$ to $k=s-1+n s$ and for $n \geq 0$ the problem solved by $\bar{Q}^{k}, \bar{R}^{k}$, and $\bar{c}_{k}=\tilde{c}_{k}$ is solved by the trivial solution $(0,0,0)$. We argue by induction on $n$. Let us look at the case $n=0$. We argue by induction on $k$. The 
problem solved by $\bar{Q}^{1}, \bar{R}^{1}$, and $\bar{c}_{1}$ is

$$
\left\{\begin{array}{l}
-\mu \bar{c}_{0} \partial_{\sigma}^{2} \bar{Q}^{1}=\bar{c}_{1} \delta_{0}+\mu \bar{c}_{1} \partial_{\sigma}^{2} \bar{Q}^{0} \\
-\mu \bar{c}_{0} \partial_{\sigma}^{2} \bar{R}^{1}+\bar{R}^{1}=\mu \bar{c}_{1} \partial_{\sigma}^{2} \bar{R}^{0} \\
\bar{Q}^{1}( \pm 1)=\bar{R}^{1}( \pm 1) \\
\partial_{\sigma} \bar{Q}^{1}( \pm 1)=\partial_{\sigma} \bar{R}^{1}( \pm 1) \\
\int_{-1}^{1} \bar{Q}^{1}(\sigma) \mathrm{d} \sigma+\frac{\bar{c}_{1}}{\mu}=0
\end{array}\right.
$$

Now, using the fundamental problem, we see that $\bar{c}_{1} \delta_{0}+\mu \bar{c}_{1} \partial_{\sigma}^{2} \bar{Q}^{0}=0$. It is now clear that the trivial solution satisfies this problem. We can see now that for $k=2, \ldots, s-1$, $\bar{Q}^{k}, \bar{R}^{k}$, and $\bar{c}_{k}$ satisfy

$$
\left\{\begin{array}{l}
-\mu \bar{c}_{0} \partial_{\sigma}^{2} \bar{Q}^{k}=\bar{c}_{k} \delta_{0}+\mu \bar{c}_{k} \partial_{\sigma}^{2} \bar{Q}^{0}=0 \\
-\mu \bar{c}_{0} \partial_{\sigma}^{2} \bar{R}^{k}+\bar{R}^{k}=\mu \bar{c}_{k} \partial_{\sigma}^{2} R b^{0} \\
\bar{Q}^{k}( \pm 1)=\bar{R}^{k}( \pm 1) \\
\partial_{\sigma} \bar{Q}^{k}( \pm 1)=\partial_{\sigma} \bar{R}^{k}( \pm 1) \\
\int_{-1}^{1} \bar{Q}^{k}(\sigma) \mathrm{d} \sigma+\frac{\bar{c}_{k}}{\mu}=0
\end{array}\right.
$$

which is again satisfied by the trivial $(0,0,0)$ solution. Now let us assume that we have proved our result for $n=0, \ldots, p-1$. We prove that $\bar{Q}^{1+p s}, \bar{R}^{1+p s}$, and $\bar{c}_{1+p s}$ are trivial. The other cases can be deduced by induction in the same fashion as in the $n=0$ case. The equation solved by $\bar{Q}^{1+p s}$ is (5.4a) with $k=1+p s \geq s$, which is

$$
-\mu \sum_{k^{\prime}}^{1+p s} \bar{c}_{k^{\prime}} \partial_{\sigma}^{2} \bar{Q}^{1+p s-k^{\prime}}+\partial_{\sigma} \bar{Q}^{1+p s-s}=\bar{c}_{1+p s} \delta_{0} .
$$

Now $\bar{Q}^{1+(p-1) s}$ is identically 0 by hypothesis, so $\partial_{\sigma} \bar{Q}^{1+p s-s}$ is zero. And if we consider the sum, the potentially nonzero coefficients are $\bar{c}_{0}, \bar{c}_{s}, \ldots, \bar{c}_{p s}$, and also $\bar{c}_{1+p s}$. But for $k \geq 1, \bar{c}_{k s}$ is multiplied by $\partial_{\sigma}^{2} \bar{Q}^{1+(p-k) s}$, which is zero by hypothesis. So the equation on $\bar{Q}^{1+p s}$ is really only

$$
-\mu \bar{c}_{0} \partial_{\sigma}^{2} \bar{Q}^{1+p s}=\bar{c}_{1+p s} \delta_{0}+\mu \bar{c}_{1+p s} \partial_{\sigma}^{2} \bar{Q}^{0}=0
$$

when using the fundamental problem. In the same way the equation in $\bar{R}^{1+p s}$ reduces to

$$
-\mu \bar{c}_{0} \partial_{\sigma}^{2} \bar{R}^{1+p s}+\bar{R}^{1+p s}=\mu \bar{c}_{1+p s} \partial_{\sigma}^{2} \bar{R}^{0}
$$

which means that once again we can take $\bar{Q}^{1+p s}=0, \bar{R}^{1+p s}=0$, and thus $\bar{c}_{1+p s}=0$. Finally, only the multiples of $s$ are potentially nonzero, and we can minimize the number of equations by taking $s=1$. 
Remark 2. The fact that we use the analytical solution of the fundamental problem seems contradictory to our intent not to compute solutions explicitly. But what we want to avoid is computing the analytical solution of the exact initial problem. We believe that it will be within our reach to do exact computations on the fundamental problem even in several space dimensions.

Lemma 5.5. If $\tilde{c}_{0}=0$ and $2 l \geq s$, then $\mu<1 / 2$, and one can take $l=1$ and $s=2$.

Proof. Since $\tilde{c}_{0}=0$, we know that we must have $c_{l} \neq 0$. Once again we look at the fundamental problem. Since $\bar{R}^{0}$ and $R^{0}$ are both zero, $\bar{Q}^{0}$ must vanish on the boundary of $[-1,1]$. Finally, with $\tilde{c}_{0}=0, \bar{Q}^{0}$ must be of integral 1 . Now there are two possibilities. If $2 l>s$, then the first nontrivial equation featuring $\bar{Q}^{0}$ is for (5.4a) with $k=s$ and is simply $\partial_{\sigma} \bar{Q}^{0}=0$. This leads to a contradiction because $\bar{Q}^{0}$ would then be a constant which, given the boundary conditions, is 0 , which is incompatible with the constraint. Thus we must have $2 l=s$.

Then the equation on $\bar{Q}^{0}$ comes from (5.4a), $k=2 l$, and sums up to system (3.4). Its solution is given by (3.5), and in particular we have $\mu<1 / 2$. We still have to see what $l$ or $s$ is. The first boundary profile to be nonzero is $R^{l}$, and it gives boundary conditions to the problem of $\bar{Q}^{l}$. It is then easy to see that to minimize the number of nontrivial problems one can take $l=1$ and thus $s=2$.

Lemma 5.6. If $\tilde{c}_{0}=0$ and $2 l+1 \leq s$, then

1. $\mu=1 / 2$,

2. $l \geq 2$ and $2 s=5 l$, and

3. one can take $l=2$ and $s=5$.

Proof. Under the conditions of the lemma, the fundamental problem is system (3.7). We have the fundamental solution given by (3.8), but not $c_{l}$, and the most difficult part is to find the right boundary layer size and expansion scale. To do that, we compute the first nontrivial boundary layer profile. We take $k=2 l$ in (5.4b) (at this point it does not matter if $2 l$ is lower or greater than $s-l$ because all the $R^{k}$ are zero for $k<l)$ and boundary condition $(5.4 \mathrm{~g})$ to find that $R^{l}$ satisfies

$$
\left\{\begin{array}{l}
-\mu c_{l} \partial_{z}^{2} R^{l}( \pm 1, \cdot)+R^{l}( \pm 1, \cdot)=0 \\
\partial_{z} R^{l}(-1,0)=1 \\
\partial_{z} R^{l}(1,0)=1
\end{array}\right.
$$

whose solution is $R^{l}( \pm 1, z)=\sqrt{\mu c_{l}} \exp \left(-z / \sqrt{\mu c_{l}}\right)$.

Now let us examine (5.4a) obeyed by $\bar{Q}^{p}$. The first equation to have $\bar{Q}^{p}$ in a nontrivial manner is $k=2 l+p$ because the first nontrivial $\tilde{c}_{k}$ is for $k=2 l$. In this equation there are two possible "external forces." First there are the boundary conditions (5.4e). The first nonzero boundary condition for a $\bar{Q}^{k}$ is $k=l$. The second "external force" is the term of the form $\partial_{\sigma}^{k-s}$. The first $\bar{Q}^{k}$ to have a nontrivial term of this form is when $k=s$, which is for the equation introducing $\bar{Q}^{s-2 l}$.

It is easy to see by induction that for $1 \leq k \leq \min (s-2 l, l)-1$ the $\bar{Q}^{k}$ are zero because they have no "external force" and they actually follow a linear equation (also (5.4h) gives the constraint $\int_{-1}^{1} \bar{Q}^{k}(\sigma) \mathrm{d} \sigma=0$ as long as $1 \leq k \leq 2 l-1$, which is of course satisfied by a trivial $\left.\bar{Q}^{k}\right)$.

If we had $l<s-2 l$, then we would have $2 l+l \leq s-1$, and $\bar{Q}^{l}$ would satisfy the 
following problem:

$$
\left\{\begin{array}{l}
-\mu c_{l} \partial_{\sigma}^{2} \bar{Q}^{l}=0, \\
\bar{Q}^{l}( \pm 1)=R^{l}( \pm 1,0), \\
\int_{-1}^{1} \bar{Q}^{l}(\sigma) \mathrm{d} \sigma=0 .
\end{array}\right.
$$

Since we have $R^{l}(1,0)=R^{l}(-1,0)=\sqrt{\mu c_{l}}$, we clearly have $\bar{Q}^{l}(\sigma)=\sqrt{\mu c_{l}}$, but this leads to a contradiction between $c_{l} \neq 0$ and $\int_{-1}^{1} \bar{Q}^{l}(\sigma) \mathrm{d} \sigma=0$. This means we must have $s-2 l \leq l$, and thus the first term to have an external force is $\bar{Q}^{s-2 l}$, whose equation is given by (5.4a) with $k=s$. This leads to $\bar{Q}^{s-2 l}$ to satisfy the problem

$$
\begin{cases}-\mu c_{l} \partial_{\sigma}^{2} \bar{Q}^{s-2 l}+\partial_{\sigma} \bar{Q}^{0}=0, & \\ \bar{Q}^{s-2 l}( \pm 1)=R^{l}( \pm 1,0) & \text { if } s-2 l=l, \\ \bar{Q}^{s-2 l}( \pm 1)=0 & \text { if } s-2 l<l, \\ \int_{-1}^{1} \bar{Q}^{s-2 l}(\sigma) \mathrm{d} \sigma=0 . & \end{cases}
$$

We now prove that $s-2 l$ cannot be $l$. If it were, we could decompose $\bar{Q}^{s-2 l}$ into a sum $A+B$ by linearity where we have

$$
\left\{\begin{array}{l}
-\mu c_{l} \partial_{\sigma}^{2} A=0 \\
A( \pm 1)=R^{l}( \pm 1,0)
\end{array}\right.
$$

and

$$
\left\{\begin{array}{l}
-\mu c_{l} \partial_{\sigma}^{2} B=-\partial_{\sigma} \bar{Q}^{0}, \\
B( \pm 1)=0 .
\end{array}\right.
$$

Since $R^{l}(1,0)=R^{l}(-1,0)=\sqrt{\mu c_{l}}$, we once again have $A=\sqrt{\mu c_{l}}$. Now since we have $\partial_{\sigma} \bar{Q}^{0}$ odd, we have that $B$ is the sum of the odd primitive of $1 /\left(\mu c_{l}\right) \bar{Q}^{0}$ and of an affine function and considering the boundary condition $B$ is in fact odd. Together these lead to a contradiction since we should have

$$
\begin{aligned}
0=\int_{-1}^{1} \bar{Q}^{s-2 l}(\sigma) \mathrm{d} \sigma & =\int_{-1}^{1} A(\sigma) \mathrm{d} \sigma+\int_{-1}^{1} B(\sigma) \mathrm{d} \sigma \\
& =2 \sqrt{\mu c_{l}},
\end{aligned}
$$

with $c_{l} \neq 0$. Thus $s-2 l<l$. From $2 l+1 \leq s \leq 3 l-1$ we get $l \geq 2$. And we have that the problem satisfied by $\bar{Q}^{s-2 l}$ is well-posed. We actually need $\bar{Q}^{s-2 l}$. One can prove that it is the function

$$
\sigma \mapsto \begin{cases}\frac{1}{c_{l}} \sigma(\sigma+1) & \text { if } \sigma \in[-1,0], \\ \frac{1}{c_{l}} \sigma(1-\sigma) & \text { if } \sigma \in[-1,0] .\end{cases}
$$

But $c_{l}$ is still not defined! We thus push the study to the following nonzero term. From the "external force" point of view, the boundary condition still drives $\bar{Q}^{l}$ first. 
For the derivative it is $\partial_{\sigma} \bar{Q}^{s-2 l}$, which appears first in (5.4a) with $k=2 s-2 l$, which gives the equation of $\bar{Q}^{2 s-4 l}$.

What we prove now is that both "external forces" need to be active simultaneously to have a well-posed problem. This leads to $l=2 s-4 l$, which is equivalent to $2 s=5 l$. If $l<2 s-4 l$, then the problem satisfied by $\bar{Q}^{l}$ is now

$$
\left\{\begin{array}{l}
-\mu c_{l} \partial_{\sigma}^{2} \bar{Q}^{l}=\mu c_{4 l-s} \partial_{\sigma}^{2} \bar{Q}^{s-2 l} \\
\bar{Q}^{l}( \pm 1)=R^{l}( \pm 1,0) \\
\int_{-1}^{1} \bar{Q}^{l}(\sigma) \mathrm{d} \sigma=0 .
\end{array}\right.
$$

Since $\bar{Q}^{s-2 l}$ is odd, this "external force" does not contribute to the integral constraint. Moreover, we can still lift the boundary condition with the constant function $\sqrt{\mu c_{l}}$. This leads to a contradiction between the integral vanishing and $c_{l}$ being nonzero. On the other hand, if $2 s-4 l<l$, then the problem solved by $\bar{Q}^{2 s-4 l}$ is

$$
\left\{\begin{array}{l}
-\mu c_{l} \partial_{\sigma}^{2} \bar{Q}^{2 s-4 l}=\mu c_{s-l} \partial_{\sigma}^{2} \bar{Q}^{s-2 l}-\partial_{\sigma} \bar{Q}^{s-2 l}, \\
\bar{Q}^{2 s-4 l}( \pm 1)=0, \\
\int_{-1}^{1} \bar{Q}^{2 s-4 l}(\sigma) \mathrm{d} \sigma=0 .
\end{array}\right.
$$

Once again, $\partial_{\sigma}^{2} \bar{Q}^{s-2 l}$ being odd, $\int_{-1}^{1} \bar{Q}^{2 s-4 l}(\sigma) \mathrm{d} \sigma$ by linearity is really only $\int_{-1}^{1} C(\sigma) \mathrm{d} \sigma$, where $C$ solves

$$
\left\{\begin{array}{l}
-\mu c_{l} \partial_{\sigma}^{2} C=-\partial_{\sigma} \bar{Q}^{s-2 l}, \\
C( \pm 1)=0 .
\end{array}\right.
$$

If we multiply the equation for $C$ by $\left(\sigma^{2}-1\right) / 2$ and integrate twice by parts, we find

$$
\mu c_{l} \int_{-1}^{1} C(\sigma) \mathrm{d} \sigma=-\int_{-1}^{1} \bar{Q}^{s-2 l}(\sigma) \sigma \mathrm{d} \sigma .
$$

Now using the expression of $\bar{Q}^{s-2 l}$ one finds $\int_{-1}^{1} C(\sigma) \mathrm{d} \sigma=1 /\left(3 c_{l}^{2}\right)$, and this cannot be 0 . We then necessarily have $2 s-4 l=l$, that is, $2 s=5 l$. It is left to the reader to see that $s=5$ and $l=2$ lead to the minimum of nontrivial profiles. One also finds that $c_{l}$ satisfies the equation

$$
2 \sqrt{\mu c_{l}}-\frac{1}{3 c_{l}^{2}}=0,
$$

which leads to $c_{l}=1 /(3 \sqrt{2})^{2 / 5}$.

\section{Justification of the formal expansions.}

6.1. Reformulation as a two parameter problem. We shall now give a rigorous proof that the behavior of $(4.1 \mathrm{a})-(4.1 \mathrm{~h})$ is the one described by Theorem 4.1. For this, we first rewrite the system in new variables. We then explain the argument of the proof using some technical assumptions. We finally check that these assumptions 
are true (unique solvability of the fundamental problem, analyticity, and derivatives of the implicit function).

Reformulation through new parameters. A crucial step in the analysis is to rewrite the system with new variables: instead of having $y$ and $\phi$, we set

$$
a=\frac{y}{\phi} \quad \text { and } \quad b=\sqrt{\phi} .
$$

Let us remark that this kind of change of variable was also necessary in our previous paper [13] in order to study the behavior near the singularity $(y, \phi)=(0,0)$. However, $y$ and $\phi$ have clear physical meaning (the first is a shear rate, and the second is the fluidity). We then define from the solution $p$ the functions

$$
\begin{aligned}
q & =p_{\uparrow}[-1,1], & & \\
r_{d}(\theta) & =p(1+b \theta) & & \text { for } \theta>0, \\
r_{g}(\theta) & =p(-1-b \theta) & & \text { for } \theta>0 .
\end{aligned}
$$

In these variables (1.1) can be written as

$$
\begin{array}{lr}
-\partial_{\sigma}^{2} q+a \partial_{\sigma} q=\frac{1}{\mu} \delta_{0} & \text { in }]-1,1[, \\
-\partial_{\theta}^{2} r_{g}-a b \partial_{\theta} r_{g}+r_{g}=0 & \text { in }] 0, \infty[, \\
-\partial_{\theta}^{2} r_{d}+a b \partial_{\theta} r_{d}+r_{d}=0 & \text { in }] 0, \infty[ \\
r( \pm \infty)=0, & \\
q \geq 0 & \\
r \geq 0 & \\
r_{g}(0)=q(-1), & \\
r_{d}(0)=q(1), & \\
-\partial_{\theta} r_{g}(0)=b \partial_{\sigma} q(-1), & \\
\partial_{\theta} r_{d}(0)=b \partial_{\sigma} q(1), & \\
b \int_{0}^{+\infty} r_{g}(\theta) \mathrm{d} \theta+b \int_{0}^{+\infty} r_{d}(\theta) \mathrm{d} \theta+\int_{-1}^{1} q(\sigma) \mathrm{d} \sigma=1 . &
\end{array}
$$

A major advantage of this formulation is that the reformulated problem no longer is of a singularly perturbed nature. This is the deeper reason why in the formal expansion of the previous section there was never any true two parameter series; either all the $R^{k}$ or all the $\bar{R}^{k}$ were zero. In several dimensions, the singularly perturbed nature of the problem cannot be removed by a mere rescaling of the independent variable, and this will complicate the analysis. In particular, analyticity of the solution with respect to $a$ and $b$ as shown below cannot be expected.

Proof of Theorem 4.1. In terms of the two parameter expansion, the three cases encountered in the previous section are as follows:

1. $\mu>1 / 2: a \rightarrow 0, b \rightarrow b_{0}>0$.

2. $\mu<1 / 2: b \rightarrow 0, a \rightarrow a_{0}>0$.

3. $\mu=1 / 2$ : Both $a$ and $b$ tend to 0 , and $b$ is of the same order as $a^{2}$.

The structure of our argument essentially proceeds as follows: First we show that if either $a$ or $b$ is close to 0 , we can solve the system (6.1a)-(6.1j) uniquely for $q, r_{d}$, and $r_{g}$ as functions of $\mu, a$, and $b$. The remaining equation $(6.1 \mathrm{k})$ is then of the form $F(\mu, a, b)=0$. The function $F$ depends analytically on its arguments. 
Case $\mu>1 / 2$. We can show that $\partial F / \partial b\left(0, b_{0}, \mu\right) \neq 0$; hence we solve the equation $F=0$ for $b: b=g(\mu, a)$. In terms of $y$ this reads $\sqrt{\phi(y)}=g(\mu, y / \phi(y))$. We can now use the implicit function theorem again to solve for $\phi$ as an analytical function of $y$.

Case $\mu<1 / 2$. We can show that $\partial F / \partial a\left(a_{0}, 0, \mu\right) \neq 0$ and hence solve for $a$ : $a=g(\mu, b)$. In terms of the original variables, this reads $y=\phi(y) g(\mu, \sqrt{\phi(y)})$. We can now apply another implicit function argument to solve for $\sqrt{\phi(y)}$ as an analytical function of $\sqrt{y}$; the leading order term is $\sqrt{\phi(y)} \sim \sqrt{y / g(\mu, 0)}=\sqrt{y / a_{0}}$.

Case $\mu=1 / 2$. For $\mu$ near $1 / 2$, we can solve $F$ uniquely for $\mu: \mu-1 / 2=g(a, b)$. This confirms that $\mu=1 / 2$ is the only case in which $a=b=0$. We shall show that, to leading order, $g(a, b)=c_{1} b-c_{2} a^{2}$ with positive constants $c_{1}$ and $c_{2}$. Thus, at $\mu=1 / 2$, we have a balance of $b$ and $a^{2}$. If we fix $\mu$ at $1 / 2$, we can solve for $b$ in terms of $a$ :

$$
b=\gamma_{2} a^{2}+\gamma_{3} a^{3}+\cdots, \quad \gamma_{2}=c_{2} / c_{1} .
$$

Now we substitute $b=\beta y^{2 / 5}$, leading to $a=\beta^{-2} y^{1 / 5}$. We find the following new equation for $\beta$ :

$$
\beta=\gamma_{2} / \beta^{4}+\gamma_{3} y^{1 / 5} / \beta^{6}+\cdots
$$

Another implicit function argument shows that $\beta$ is an analytical function of $y^{1 / 5}$, with leading order term $\beta \sim\left(\gamma_{2}\right)^{1 / 5}$.

6.2. The fundamental problems. We are now exhibiting the three fundamental solutions we will perturb; that is, we will uniquely solve (6.1a)-(6.1j) for specific sets of the parameters $(\mu, a, b)$. Of course, we will find the three fundamental problems (3.4), (3.2), and (3.7) rewritten in the new variables (without satisfying the integral constraint yet).

Set $a=0$ in the previous equations, and let us find the $\mu$ and $b$ that satisfy the equations. We can solve the ODEs and use the various constraints to set the integration constants. This leads to

$$
\begin{aligned}
& q^{\mu, 0, b}(\sigma)= \begin{cases}\frac{1}{2 \mu}(\sigma+1)+\frac{b}{2 \mu} & \text { if } \sigma \in]-1,0[, \\
\frac{1}{2 \mu}(1-\sigma)+\frac{b}{2 \mu} & \text { if } \sigma \in] 0,1[,\end{cases} \\
& r_{g}^{\mu, 0, b}(\theta)=\frac{b}{2 \mu} \exp (-\theta) \\
& r_{d}^{\mu, 0, b}(\theta)=\frac{b}{2 \mu} \exp (-\theta) .
\end{aligned}
$$

This indeed gives back (3.2) when one sets $b=\sqrt{\mu \bar{c}_{0}}$.

On the other hand set $b=0$. Then the equations of $q$ on one hand and $r_{g}, r_{d}$ on the other hand decouple. We can set $r_{g}=r_{d}=0$ by linearity, and this makes the problem set on the interval $[-1,1]$ only, with homogeneous Dirichlet boundary conditions. This leads to

$$
\begin{aligned}
q^{\mu, a, 0}(\sigma) & = \begin{cases}\frac{1-\exp (-a)}{\mu a(\exp (a)-\exp (-a))}(\exp (a(\sigma+1))-1) & \text { if } \sigma \in]-1,0[, \\
\frac{\exp (a)-1}{\mu a(\exp (a)-\exp (-a))}(1-\exp (a(\sigma-1))) & \text { if } \sigma \in] 0,1[,\end{cases} \\
r_{g}^{\mu, a, 0} & =0 \\
r_{d}^{\mu, a, 0} & =0 .
\end{aligned}
$$

Copyright $@$ ㅇ by SIAM. Unauthorized reproduction of this article is prohibited. 
We find (3.5) again when $a=1 /\left(\mu c_{1}\right)$. Finally, taking the limit $b \rightarrow 0$ in the first case or $a \rightarrow 0$ in the second leads to the same solution for $a=b=0$,

$$
q^{\mu, 0,0}(\sigma)= \begin{cases}\frac{1}{2 \mu}(\sigma+1) & \text { if } \sigma \in]-1,0[, \\ \frac{1}{2 \mu}(1-\sigma) & \text { if } \sigma \in] 0,1[,\end{cases}
$$

which gives back (3.8) when we set $\mu=1 / 2$.

6.3. Unique solvability of the ODE system. We define the weighted Sobolev space

$$
H_{\varepsilon}^{s}(0, \infty)=\left\{u \mid \exp (\varepsilon \theta) u(\theta) \in H^{s}(0, \infty)\right\} .
$$

We fix a small $\varepsilon>0$, and we seek solutions $\left(q, r_{d}, r_{g}\right)$ in $H^{1}(-1,1) \times\left(H_{\varepsilon}^{1}(0, \infty)\right)^{2}$. We can get rid of the weight $\exp (\varepsilon \theta)$ by setting $r_{g, d}=\exp (-\varepsilon \theta) s_{g, d}$. Moreover, we isolate the singularity of $q$ at the origin by setting $\tilde{q}(\sigma)=q(\sigma)-(1-|\sigma|) /(2 \mu)$. This leads to the new system

$$
\begin{array}{lc}
-\partial_{\sigma}^{2} \tilde{q}+a \partial_{\sigma} \tilde{q}=\frac{a}{2 \mu} \operatorname{sign} \sigma & \text { in }]-1,1[, \\
-\partial_{\theta}^{2} s_{g}-(a b-2 \varepsilon) \partial_{\theta} s_{g}+\left(1-\varepsilon^{2}+a b \varepsilon\right) s_{g}=0 & \text { in }] 0, \infty[, \\
-\partial_{\theta}^{2} s_{d}+(a b-2 \varepsilon) \partial_{\theta} s_{d}+\left(1-\varepsilon^{2}-a b \varepsilon\right) s_{d}=0 & \text { in }] 0, \infty[, \\
s_{g}(0)=\tilde{q}(-1), & \\
s_{d}(0)=\tilde{q}(1), & \\
-\partial_{\theta} s_{g}(0)+\varepsilon s_{g}(0)=b \partial_{\sigma} \tilde{q}(-1)+b /(2 \mu), & \\
\partial_{\theta} s_{d}(0)-\varepsilon s_{d}(0)=b \partial_{\sigma} \tilde{q}(1)-b /(2 \mu) . &
\end{array}
$$

We now look for solutions in the function space

$$
X=\left\{\left(\tilde{q}, s_{d}, s_{g}\right) \in H^{2}(-1,1) \times\left(H^{2}(0, \infty)\right)^{2} / s_{g}(0)-\tilde{q}(-1)=0, s_{d}(0)-\tilde{q}(1)=0\right\} .
$$

Define $Y$ as

$$
Y=L^{2}(-1,1) \times\left(L^{2}(0, \infty)\right)^{2} \times(\mathbb{R})^{2} .
$$

We define the operator $\mathcal{L}_{\varepsilon, a, b}$ from $X$ to $Y$ by

$$
\begin{aligned}
\mathcal{L}_{\varepsilon, a, b}\left(\tilde{q}, s_{d}, s_{g}\right)= & \left(-\tilde{q}^{\prime \prime}+a \tilde{q}^{\prime},\right. \\
& -s_{g}^{\prime \prime}-(a b-2 \varepsilon) s_{g}^{\prime}+\left(1-\varepsilon^{2}+a b \varepsilon\right) s_{g}, \\
& -s_{d}^{\prime \prime}+(a b-2 \varepsilon) s_{d}^{\prime}+\left(1-\varepsilon^{2}-a b \varepsilon\right) s_{d}, \\
& -s_{g}^{\prime}(0)+\varepsilon s_{g}(0)-b \tilde{q}^{\prime}(-1), \\
& \left.s_{d}^{\prime}(0)-\varepsilon s_{d}(0)-b \tilde{q}^{\prime}(1)\right) .
\end{aligned}
$$

Let us note that $E=\mathbf{R} \times\{0\} \cup\{0\} \times \mathbf{R}_{+}$. We claim the following proposition. Proposition 6.1.

- For every $\left(a_{0}, b_{0}\right) \in E$ there exist an $\varepsilon_{0}>0$ and a neighborhood $\mathcal{V}$ of $\left(a_{0}, b_{0}\right)$ in $\mathbf{R} \times \mathbf{R}_{+}$such that for any $(\varepsilon, a, b) \in\left[0, \varepsilon_{0}\left[\times \mathcal{V}\right.\right.$ the operator $\mathcal{L}_{\varepsilon, a, b}$ is invertible.

- The mapping $(\mu, a, b) \mapsto\left(q^{\mu, a, b}, r_{g}^{\mu, a, b}, r_{d}^{\mu, a, b}\right)$ is analytic.

Copyright (c) by SIAM. Unauthorized reproduction of this article is prohibited. 
Proof. For the proof of the first point, it clearly suffices to consider $\varepsilon=0$ and either $a=0$ or $b=0$; the rest follows from analytic perturbation theory (see, for instance, Kato [12, Chap. VII, p. 365]). Indeed, because we removed the singularity at $0, \mathcal{L}_{\varepsilon, a, b}$ is a bounded operator from $X$ to $Y$. Moreover, for any $\left(\tilde{q}, s_{d}, s_{g}\right)$ in $X$ and $f$ in $Y^{*},\left\langle f, \mathcal{L}_{\varepsilon, a, b}\left(\tilde{q}, s_{d}, s_{g}\right)\right\rangle_{Y^{*}, Y}$ is a polynomial in $(\varepsilon, a, b)$, and thus $\mathcal{L}_{\varepsilon, a, b}$ is analytic as an operator.

If $\varepsilon=b=0$, the problems for $\tilde{q}, s_{g}$, and $s_{d}$ decouple, and the decoupled problems are easy to analyze: we solve elliptic ODEs with inhomogeneous Neumann boundary conditions for $s_{g}$ and $s_{d}$ and then use the values $s_{g}(0)$ and $s_{d}(0)$ in the boundary conditions for $\tilde{q}$. Now, let $\varepsilon=a=0, b>0$. Note that for $\left(\tilde{q}, s_{d}, s_{g}\right) \in X$ we have the following Poincaré-type estimate:

$$
\int_{-1}^{1} \tilde{q}(\sigma)^{2} \mathrm{~d} \sigma \leq 4 \int_{-1}^{1}\left(\tilde{q}^{\prime}(\sigma)\right)^{2} \mathrm{~d} \sigma+4 \int_{0}^{+\infty}\left(s_{g}^{\prime}(\theta)\right)^{2}+s_{g}(\theta)^{2} \mathrm{~d} \theta .
$$

It is then easily checked that the following elliptic estimate is true:

$$
\int_{-1}^{1}\left(\tilde{q}^{\prime}(\sigma)\right)^{2} d \sigma+\frac{1}{b} \int_{0}^{\infty}\left(s_{g}^{\prime}(\theta)\right)^{2}+s_{g}(\theta)^{2}+\left(s_{d}^{\prime}(\theta)\right)^{2}+s_{d}(\theta)^{2} \mathrm{~d} \theta \leq C\|f\|_{Y}^{2} .
$$

The operator $\mathcal{L}_{0,0, b}$ is then invertible by standard arguments using the Lax-Milgram theorem and regularity of elliptic operators.

The second point uses the fact that $\mathcal{L}_{\varepsilon, a, b}^{-1}$, as a function of $a$ and $b$, is analytic, and so is

$$
(\mu, a, b) \mapsto\left(\frac{a}{2 \mu} \operatorname{sign} \sigma, 0,0,0,0, \frac{b}{2 \mu},-\frac{b}{2 \mu}\right),
$$

and finally so are the various changes of variables.

6.4. Derivatives of $\boldsymbol{F}$. We now set

$$
F(\mu, a, b)=\int_{-1}^{1} q(\sigma) d \sigma+b \int_{0}^{\infty} r_{g}(\theta) d \theta+b \int_{0}^{\infty} r_{d}(\theta) d \theta
$$

where $q, r_{d}$, and $r_{g}$ have been determined as solutions of the ODE system, as discussed in the previous subsection.

If $a=0$, we compute, using (6.2),

$$
F(\mu, 0, b)=\frac{b+b^{2}}{\mu}+\frac{1}{2 \mu} .
$$

A nonnegative solution $b_{0}$ of the equation $F\left(\mu, 0, b_{0}\right)=1$ exists if and only if $\mu \geq 1 / 2$. Moreover, we find

$$
\begin{aligned}
& \frac{\partial F}{\partial b}\left(\mu, 0, b_{0}\right)=\frac{1+2 b_{0}}{\mu}>0, \\
& \frac{\partial F}{\partial \mu}\left(\mu, 0, b_{0}\right)=-\frac{F\left(\mu, 0, b_{0}\right)}{\mu}=-\frac{1}{\mu}<0 .
\end{aligned}
$$

If $b=0$, we calculate, using (6.3),

$$
F(\mu, a, 0)=\frac{\tanh (a / 2)}{a \mu} .
$$

Copyright ( $)$ by SIAM. Unauthorized reproduction of this article is prohibited. 
As $a$ varies, this function decreases monotonically from a limit of $1 /(2 \mu)$ at $a=0$ to zero as $a \rightarrow \infty$. Hence the equation $F\left(\mu, a_{0}, 0\right)=1$ has a solution $a_{0}$ if and only if $\mu \leq 1 / 2$. The derivative $\partial F / \partial a(\mu, a, 0)$ is negative for $a>0$, but $\partial F / \partial a(\mu, 0,0)=0$. Moreover, we find

$$
\frac{\partial^{2} F}{\partial a^{2}}(\mu, 0,0)=-\frac{1}{12 \mu}<0
$$

This verifies all the sign properties of derivatives of $F$ claimed above.

Acknowledgment. Julien Olivier would like to thank D. Bresch for his involvement and support.

\section{REFERENCES}

[1] P. Angot, C. H. Bruneau, and P. Fabrie, A penalization method to take into account obstacles in incompressible viscous flows, Numer. Math., 81 (1999), pp. 497-520.

[2] J.-P. Bouchaud, A. Comtet, And C. Monthus, On a dynamical model of glasses, J. Phys. I France, 5 (1995), pp. 1521-1526.

[3] F. Boyer And P. FABrie, Éléments d'Analyse pour l'Étude de Quelques Modèles d'Écoulements Visqueux Incompressibles, Mathématiques et Applications (Berlin) 52, Springer-Verlag, Berlin, 2006.

[4] E. CAncès, I. CATTO, AND Y. Gati, Mathematical analysis of a nonlinear parabolic equation arising in the modelling of non-Newtonian flows, SIAM J. Math. Anal., 37 (2005), pp. $60-82$.

[5] G. Carbou, Penalization method for viscous incompressible flow around a porous thin layer, Nonlinear Anal. Real World Appl., 5 (2004), pp. 815-855.

[6] G. Carbou and P. Fabrie, Boundary layer for a penalization method for viscous incompressible flow, Adv. Differential Equations, 8 (2003), pp. 1453-1480.

[7] B. ForNET, Influence of boundary layers over the rate of convergence in a penalization method for a 1-D wave equation, Comptes Rendus Mécanique, 336 (2008), pp. 454-457.

[8] B. Fornet, A Kreiss' symmetrizer domain penalization method, Comm. Partial Differential Equations, 33 (2008), pp. 1549-1570.

[9] B. Fornet And O. GuÈs, Penalization approach to semi-linear symmetric hyperbolic problems with dissipative boundary conditions, Discrete Contin. Dyn. Syst., 23 (2009), pp. 827-845.

[10] P. HÉBraud, Propriétés mécaniques des systèmes vitreux. L'exemple des émulsions concentrées, Ph.D. thesis, Université Louis Pasteur, Strasbourg, France, 1998.

[11] P. Hébraud and F. Lequeux, Mode-coupling theory for the pasty rheology of soft glassy materials, Phys. Rev. Lett., 81 (1998), pp. 2934-2937.

[12] T. Kato, Perturbation Theory for Linear Operators, 2nd ed., Springer, Berlin, 1976.

[13] J. Olivier, Asymptotic analysis in flow curves of a model of soft glassy rheology, Z. Angew. Math. Phys., 61 (2010), pp. 445-466.

[14] G. Picard, A. Ajdari, L. Bocquet, and F. Lequeux, Simple model for heterogeneous flows of yield stress fluids, Phys. Rev. E, 66 (2002), 051501.

[15] P. Sollich, F. Lequeux, P. Hébraud, and M. Cates, Rheology of soft glassy material, Phys. Rev. Lett., 78 (1997), pp. 2020-2023.

Copyright $@$ by SIAM. Unauthorized reproduction of this article is prohibited. 